\title{
Biomasa de Cornops aquaticum (Orthoptera: Acrididae) en humedales del nordeste de Argentina
}

\author{
Luciana Irene Gallardo ${ }^{1,2}$, María Celeste Franceschini ${ }^{1,3}$, Alicia Susana Guadalupe Poi $^{1,3}$ \& \\ María Laura De Wysiecki ${ }^{4}$ \\ 1. Centro de Ecología Aplicada del Litoral- CECOAL (CCT Nordeste - CONICET- UNNE), Ruta 5 km 2.5, (3400) \\ Corrientes, Argentina; celestefranceschini@yahoo.com.ar \\ 2. Cátedra de Biología de los Invertebrados, Facultad de Ciencias Exactas y Naturales y Agrimensura-FACENA, \\ Universidad Nacional del Nordeste-UNNE, Av. Libertad 5460, (3400) Corrientes, Argentina; \\ lucianagallardo@hotmail.com \\ 3. Cátedra de Limnología, Facultad de Ciencias Exactas y Naturales y Agrimensura-FACENA, Universidad Nacional del \\ Nordeste-UNNE, (3400) Corrientes, Argentina; guadalupepoi@gmail.com \\ 4. Cátedra de Ecología de Pastizales y Sistemas Agrícolas, Facultad de Ciencias Naturales y Museo, Universidad \\ Nacional de La Plata. Centro de Estudios Parasitológicos y de Vectores (UNLP- CCT La Plata- CONICET), \\ Boulevard 120 entre 60 y 64, 1900 La Plata, Argentina; mlw@cepave.edu.ar
}

Recibido 19-II-2014. Corregido 26-VII-2014. Aceptado 25-VIII-2014.

\begin{abstract}
Biomass of Cornops aquaticum (Orthoptera: Acrididae) in wetlands of Northeast Argentina. The estimation of biomass in insect populations is a key factor to quantify the available resources and energy fluxes in ecosystems food webs. Cornops aquaticum is a common herbivore in Eichhornia plants in wetlands of Northeast Argentina. We aimed to analyse its biomass variation, related to the different grasshopper age categories populations in two host-plants: Eichhornia azurea and Eichhornia crassipes. For this, standard samplings of $C$. aquaticum populations were carried out with an entomological net of $70 \mathrm{~cm}$ diameter in two wetlands with E. azurea and E. crassipes, in Corrientes and Chaco Provinces; besides, dry weight was also obtained (directly and indirectly), and a regression model to indirectly estimate the biomass from a linear dimension measure (hind femur length) is proposed. A total of 2307 individuals were collected and separated in different age categories; their abundance and linear dimension data were obtained. The model proposed was $\ln \mathrm{DM}=\ln a+b * \ln H$ (where $\mathrm{DM}=$ dry mass, $a$ and $b$ are constants and $\mathrm{H}=$ hind femur length) $\left(\mathrm{R}^{2}=0.97\right)$. The population biomass variations of $C$. aquaticum were due to the relative abundance of each age category and the grasshopper individual dry weight. No significant differences were found between populations biomasses obtained by direct and indirect methods in E. azurea and E. crassipes floating meadows. This model made easier the C. aquaticum biomass calculation for both individuals and the population, and accelerated the processing of high number of samples. Finally, high biomass values of populations and individual age category (especially in adults) emphasize the importance of C. aquaticum as a consumer and a resource for predators on Eichhornia floating meadows food webs. Rev. Biol. Trop. 63 (1): 127-138. Epub 2015 March 01.
\end{abstract}

Key words: dry weight, Eichhornia, femur, semi-aquatic grasshopper, regression model, population abundance, water hyacinth.

En los estudios de poblaciones de insectos, la biomasa es un parámetro importante para la cuantificación de los recursos disponibles y los flujos de energía que se producen en las redes tróficas de los ecosistemas terrestres y acuáticos (Sabo, Bastow, \& Power, 2002). Además, sirve para determinar características poblacionales como producción secundaria, patrones estacionales y las relaciones tróficas existentes entre grupos funcionales (Benke, 1996).

En la mayoría de los estudios de comunidades y poblaciones de ortópteros, la abundancia tradicionalmente es expresada en relación al número de individuos (Cigliano, De Wysiecki, 
\& Lange, 2000; Gardner, \& Thompson, 2001; Squitier, \& Capinera, 2002; Torrusio, Cigliano, \& De Wysiecki, 2002; Messi, Kekeunou, \& Weise, 2006; Jannot et al., 2009; Silva, Marques, Battirola, \& Lhano, 2010a). Las estimaciones de biomasa de estos insectos son escasas y, en general, están restringidas a acridoideos de hábito semiacuático asociados a macrófitas que pueden llegar a ser invasivas en lagos y represas (Nunes, Adis, \& Nunes de Mello, 1992; Nunes, \& Adis, 1994; Adis, \& Junk, 2003; Franceschini, Adis, Poi de Neiff, \& De Wysiecki, 2007; Silva, Marques, Battirola, \& Lhano, 2010b; Braga, Nunes, Morais, \& Adis, 2012).

Los distintos valores de biomasa de los individuos determinan capacidades de consumo diferentes en las especies de insectos herbívoros asociados a una misma macrófita (Franceschini, De Wysiecki, Poi de Neiff, Galassi, \& Martínez, 2011a; Martínez, Franceschini, \& Poi, 2013). A su vez, en una misma especie de insecto herbívoro, existen variaciones en el consumo determinadas por el estado de desarrollo y la biomasa por individuo, que presenta cada categoría de edades (Franceschini et al., 2011a; Franceschini, De Wysiecki, \& Poi, 2013). La estimación directa de la biomasa puede demandar considerable tiempo de procesamiento cuando las poblaciones de insectos herbívoros presentan altas densidades, especialmente, al comparar sitios o estudiar ciclos plurianuales. Entre los métodos indirectos, las regresiones longitud-peso seco han sido muy utilizadas para estimar la biomasa a partir de una medida de dimensión lineal, por ejemplo: longitud total del cuerpo (Hodar, 1996; Benke, Huryn, Smok, \& Wallace, 1999; Miserendino, 2001; Sabo et al., 2002), ancho de la cápsula cefálica (Benke, 1996; Cressa, 1999a; Cressa, 1999b; Becker, Moretti, \& Castillo, 2009) y la distancia interocular (Becker et al., 2009). Sin embargo, deben tomarse recaudos al estimar la biomasa utilizando regresiones obtenidas para los mismos taxa en distintas regiones geográficas, ya que éstas pueden ser significativamente diferentes (Miserendino, 2001). Por lo tanto, resulta necesario disponer de regresiones longitud-peso seco para las especies de invertebrados herbívoros dominantes de los humedales presentes en el área de estudio, a efectos de proporcionar datos más precisos para la estimación de su biomasa.

Las poblaciones del acridio Cornops aquaticum Bruner, 1906, por su abundancia y biomasa, ocupan un lugar destacado entre los invertebrados herbívoros de los humedales del nordeste argentino (Franceschini et al., 2011a). Esta especie presenta un área de distribución amplia en el Neotrópico que abarca desde el sureste de México hasta el centro de Argentina y Uruguay (Adis, Bustorf, Lhano, Amedegnato, \& Nunes, 2007). Las ninfas y adultos de este acridio se alimentan de las láminas foliares de las Pontederiaceae, especialmente Eichhornia azurea (Sw.) Kunth, 1843 y Eichhornia crassipes (Mart.) Solms, 1883 (Adis, \& Junk, 2003; Lhano, Adis, Marques, \& Battirola, 2005; Franceschini, Neiff, \& Amedegnato, 2011b) y los adultos oviponen en los pecíolos de estas plantas (Center, Hill, Cordo, \& Julien, 2002). Estas macrófitas forman densas praderas flotantes que pueden llegar a cubrir gran parte de la superficie de muchos humedales de Sudamérica (Da Silva, \& Esteves, 1993; Piedade, \& Junk, 2000; Neiff, Casco, \& Poi de Neiff, 2008). En estos humedales, la abundancia y estructura de edades de la población de $C$. aquaticum tienen variaciones estacionales $\mathrm{e}$ interanuales que están en relación con las variables climáticas y con los valores de biomasa de la planta huésped (Franceschini et al., 2013).

En aéreas no nativas, $C$. aquaticum está siendo estudiado como posible agente de control biológico para la recuperación y manejo de humedales invadidos por E. crassipes (Center et al., 2002).

Los objetivos de este trabajo fueron: (1) analizar la variación estacional e interanual de la biomasa de $C$. aquaticum en relación a la abundancia de las categorías de edades de la población, comparativamente en dos plantas huéspedes: E. azurea y E. crassipes y, (2) obtener un modelo de regresión lineal para estimar la biomasa por individuo y poblacional de $C$. aquaticum de manera indirecta, a partir de una 
medida de dimensión lineal. Las hipótesis de este trabajo fueron (a) si la abundancia y la estructura poblacional de C. aquaticum varían según la estacionalidad, se espera encontrar la misma tendencia en la biomasa de la población; $\mathrm{y},(b)$ si la biomasa por individuo puede ser estimada indirectamente aplicando el modelo de regresión lineal, no se encontrarán diferencias significativas entre estos valores y los obtenidos de manera directa, a través del pesaje de los individuos.

\section{MATERIALES Y MÉTODOS}

Variaciones estacionales de la biomasa poblacional de C. aquaticum en praderas flotantes de E. azurea y E. crassipes: Los muestreos se realizaron en una laguna de la planicie de inundación del río Paraná en la provincia del Chaco $\left(27^{\circ} 26^{\prime} \mathrm{S}-58^{\circ} 51^{\prime} \mathrm{W}\right)$ y en una laguna de la cuenca del río Riachuelo alimentada exclusivamente por lluvias, en la provincia de

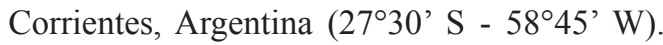
En la primera laguna, E. crassipes constituyó la planta huésped de C. aquaticum, mientras que en la segunda lo fue E. azurea; estas macrófitas no coexistieron en las lagunas estudiadas debido a que presentan diferentes requerimientos físico químicos del agua. El clima de esta área es clasificado como subtropical, con veranos y primaveras cálidos y prolongados, mientras que los inviernos son cortos y apacibles, con una frecuencia de heladas de 0.25días/año (Bruniard, 1981 y 1996).

Para cuantificar la variación estacional e interanual de la biomasa de C. aquaticum, se realizaron muestreos mensuales entre febrero 2004 y abril 2006, de 9:00 a 13:00 horas, evitando los días nublados y con lluvia. Los individuos de C. aquaticum fueron capturados utilizando una red entomológica de $70 \mathrm{~cm}$ de diámetro y $1 \mathrm{~m}$ de profundidad. La metodología empleada consistió en pasar dicha red sobre las praderas flotantes de E. azurea y E. crassipes desde una embarcación y desde tierra firme. Siguiendo a Johnson (1989), en cada fecha de muestreo se capturó un set de 50 individuos. Cuando la abundancia de C. aquaticum fue extremadamente baja imposibilitando lograr el número óptimo de individuos, se consideró el número de individuos capturados pasando la red sobre las praderas flotantes durante 30 minutos como tal, según los criterios establecidos en el Host-Insect Coevolution on Water Hyacinth Project (Adis, Lhano, Hill, Junk, Marques, \& Oberholzer, 2004; Brede, Adis, $\&$ Schneider, 2007). Previamente, se determinaron las distintas categorías de edades de la población (Franceschini et al., 2007): ninfas A (estadios I y II), ninfas B (estadios III al VI), adultos machos y adultos hembras, y se estimó la abundancia relativa de cada una de ellas. Los estadios ninfales fueron determinados utilizando la longitud del fémur posterior izquierdo (Franceschini, Capello, Lhano, Adis, \& De Wysiecki, 2005). En cada individuo se tomaron las siguientes medidas de dimensión lineal propuestas por Carbonell (2001): longitud del fémur posterior $(\mathrm{H})$, longitud desde el fastigium hasta el extremo de la tegmina (A), longitud desde el fastigium hasta el extremo del abdomen (B) y longitud de las tegminas (I).

Modelos de regresión lineal para estimar la biomasa por individuo de $C$. aquaticum a partir de una medida de dimensión lineal: En ambas lagunas se recolectaron ninfas de diferentes estadios y adultos de C. aquaticum $\left(\mathrm{N}_{\text {total }}=128\right.$ individuos) los cuales fueron colocados en un freezer durante $48 \mathrm{~h}$. Se obtuvo el peso seco de los individuos colocándolos en estufa a $100^{\circ} \mathrm{C}$ durante $72 \mathrm{~h}$. Cada individuo fue pesado en una balanza digital Shimadzu AUW 220D, colocando el material previamente en un desecador. En cada individuo se registraron las diferentes medidas de dimensión lineal propuestas por Carbonell (2001), bajo lupa binocular utilizando una regla milimetrada. Los modelos de regresión lineal para estimar la biomasa de los individuos de C. aquaticum, se obtuvieron empleando las variables peso seco por individuo y diferentes medidas de dimensión lineal. Se compararon los modelos obtenidos a fin de seleccionar la medida de dimensión lineal que mejor explicara la variabilidad del peso seco en este acridio. 
Aplicabilidad de la medida de dimensión lineal seleccionada para las estimaciones de biomasa poblacional de C. aquaticum: Para validar los resultados del modelo seleccionado, se aplicó la ecuación de regresión lineal (método indirecto) correspondiente a los datos mensuales de las poblaciones de C. aquaticum asociadas a $E$. azurea y $E$. crassipes entre enero y diciembre 2005. Estos valores fueron comparados con los obtenidos en forma directa (mediante el pesaje de los individuos), durante el período mencionado.

Las diferencias estacionales de la biomasa de la población de $C$. aquaticum en cada categoría de edades, fueron analizadas con el test de Kruskal Wallis. Las diferencias entre el peso seco por individuo de machos y hembras adultos fueron analizadas con el test de Mann Whitney (Wilcoxon). Los modelos de regresión lineal para estimar la biomasa de los individuos de C. aquaticum a partir de las distintas medidas de dimensión lineal, se obtuvieron realizando una regresión lineal simple (con las variables transformadas a $\mathrm{ln}$ ) empleando el software RStudio (versión 0.97.248). Se realizó una Prueba de Kruskal Wallis para comparar los valores de biomasa de la población de $C$. aquaticum obtenidos de manera directa, con los estimados indirectamente a partir del modelo de regresión lineal seleccionado, durante el período enero a diciembre 2005 para ambas plantas huéspedes (E. azurea y $E$. crassipes). Se consideraron significativos los valores de $\mathrm{p}<0.05$. Los análisis estadísticos se llevaron a cabo empleando el software Infostat (version 1.1).

\section{RESULTADOS}

Biomasa de la población de $C$. aquaticum en relación a la abundancia de individuos: La biomasa de la población de C. aquaticum presentó variaciones estacionales e interanuales en las praderas flotantes de E. azurea y E. crassipes (Kruskal Wallis $\mathrm{p}<0.05$ ). Los valores más altos de biomasa fueron coincidentes con los meses donde se registraron mayor número de individuos adultos en la población. La biomasa total de C. aquaticum disminuyó notablemente en los meses en que la población estaba mayormente integrada por ninfas A (Fig. 1 y Fig. 2).

Determinación directa del peso seco por individuo de la población de $C$. aquaticum: El peso seco de las ninfas y los adultos de C. aquaticum varió entre $0.0017 \mathrm{y}$ $0.1339 \mathrm{mg}$. El peso seco por individuo adulto fue significativamente mayor en las hembras (Media $=0.1031 \mathrm{mg} \pm 0.0143$ ) que en los machos (Media $=0.0554 \mathrm{mg} \pm 0.0063$ ) (Test de Mann Whitney, $\mathrm{p}<0.05 ; \mathrm{N}=57$ ). En las ninfas A, la media del peso seco de los individuos fue $(0.0034 \mathrm{mg} \pm 0.0018)$ en el estadio I, y $(0.0039 \mathrm{mg} \pm 0.0013)$ en el estadio II. El peso seco por individuo de los diferentes estadios de ninfas B fue: estadio III (Media $=0.0072 \mathrm{mg} \pm 0.0018$ ), estadio IV (Media $=0.0180 \mathrm{mg} \pm 0.0033)$ y estadio V (Media $=0.0314 \mathrm{mg} \pm 0.0092)$. Estos últimos, no mostraron diferencias significativas entre machos y hembras (Test de Kruskal Wallis, $\mathrm{p}>0.05$ ). El peso seco del VI estadio ninfal, presente sólo en hembras, fue $0.0555 \mathrm{mg} \pm 0.0161$ (Media), siendo significativamente mayor que el peso seco de los adultos machos (Test de Mann Whitney, $\mathrm{p}<0.05$ ).

Determinación indirecta del peso seco por individuo de la población de $C$. aquaticum: Los modelos de regresión lineal obtenidos a partir del peso seco por individuo de $C$. aquaticum y las distintas medidas de dimensión lineal muestran que, si bien, todas las medidas fueron significativas, la longitud del fémur posterior $(\mathrm{H})$ fue la que mejor explicó las variaciones del peso seco por individuo ya que presentó los valores más altos del Coeficiente de Determinación $\left(\mathrm{R}^{2}\right)$ y los más bajos del Criterio de Información de Akaike (AIC) y Criterio de Información Bayesiano (BIC) (Cuadro 1).

El modelo de regresión obtenido a partir de la longitud del fémur posterior y el peso seco por individuo fue $\ln P S=\ln a+b^{*} \ln H$ en su expresión linear, donde PS=peso seco, $a$ y $b$ son constantes y $\mathrm{H}=$ longitud del fémur 
A

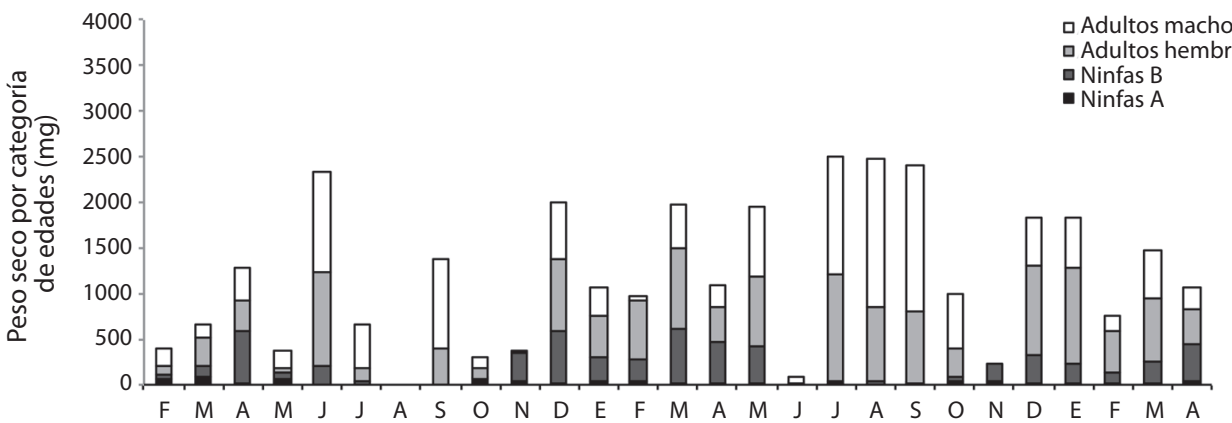

B

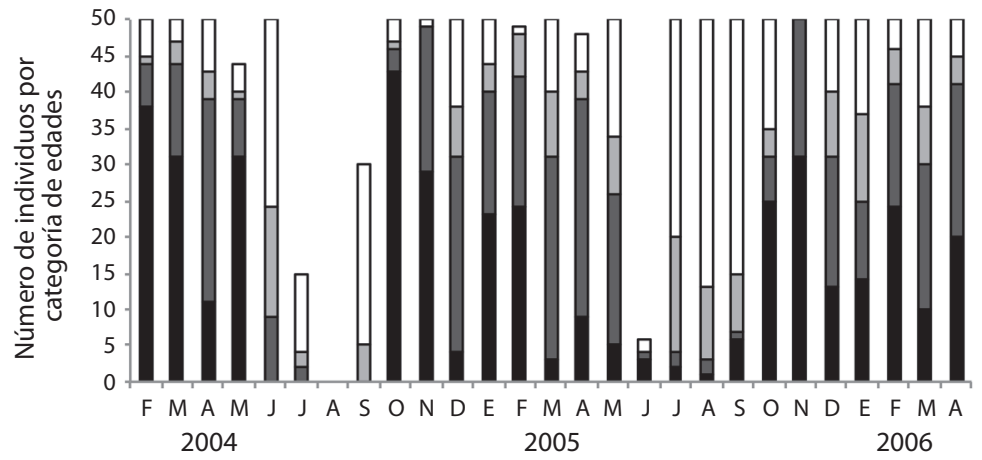

Fig. 1. (A) Biomasa de las categorías de edades de la población de Cornops aquaticum (mg de peso seco) y (B) abundancia de individuos por categoría de edades en Eichhornia azurea durante el período de muestreo.

Fig. 1. (A) Biomass of Cornops aquaticum population age categories ( $\mathrm{mg}$ of dry weight) and (B) abundance of individuals per age categories in Eichhornia azurea during the sampling period.

CUADRO 1

Estadísticas y criterios obtenidos en los análisis de regresión, utilizando distintas medidas de dimensión lineal

TABLE 1

Values of statistics and criteria obtained in the regression analysis, using different lineal dimension measures

\begin{tabular}{lcccc} 
& A & B & I & H \\
p-valor & $<0.0001$ & $<0.0001$ & $<0.0001$ & $<0.0001$ \\
$\mathrm{R}^{2}$ & 0.94 & 0.96 & 0.76 & 0.97 \\
AIC & 577.08 & 256.59 & -146.95 & 6.74 \\
BIC & 590.02 & 269.54 & -136.44 & 15.30 \\
\hline
\end{tabular}

Nivel de significancia $=\mathrm{p}$-valor $<0.05, \mathrm{R}^{2}=$ Coeficiente de determinación, $\mathrm{AIC}=$ Criterio de información de Akaike, $\mathrm{BIC}=$ Criterio de Información Bayesiano, A=Longitud desde el fastigium hasta el extremo de la tegmina, B=Longitud desde el fastigium hasta el extremo del abdomen, $\mathrm{I}=$ Longitud de la tegmina, $\mathrm{H}=$ Longitud del fémur posterior.

Significance level=p-value $<0.05, \mathrm{R}^{2}=$ Coeficient of Determination, $\mathrm{AIC}=$ Akaike's Information Criterion, $\mathrm{BIC}=\mathrm{Bayesian}$ information criterion, $\mathrm{A}=$ Lenght from the fastigium to the tegmina, $\mathrm{B}=$ Lenght from the fastigium to the end of the abdomen, $\mathrm{I}=$ Tegmina length, $\mathrm{H}=$ hind femur length. 


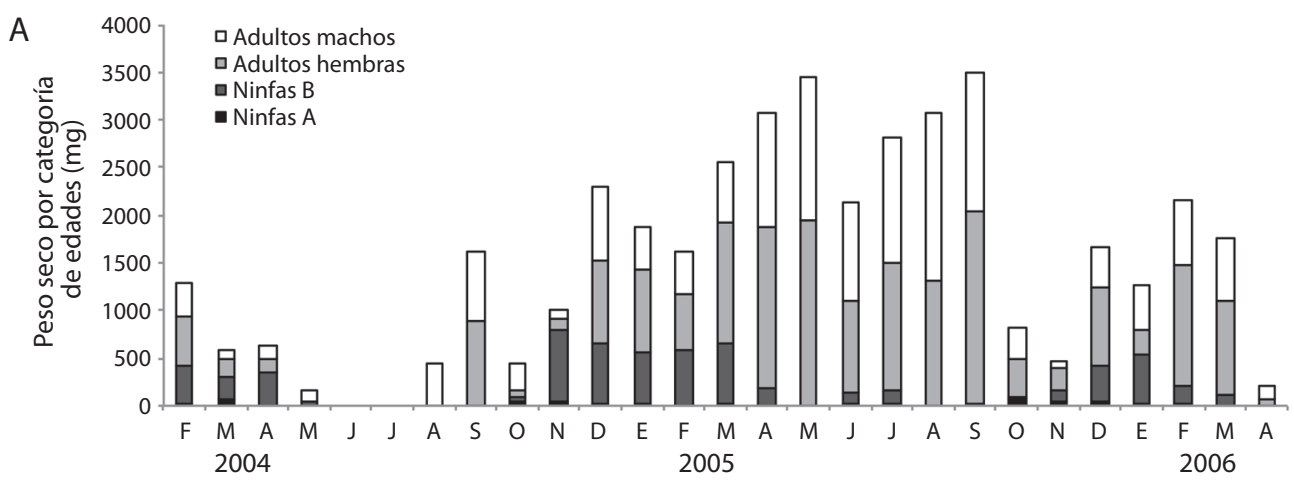

B

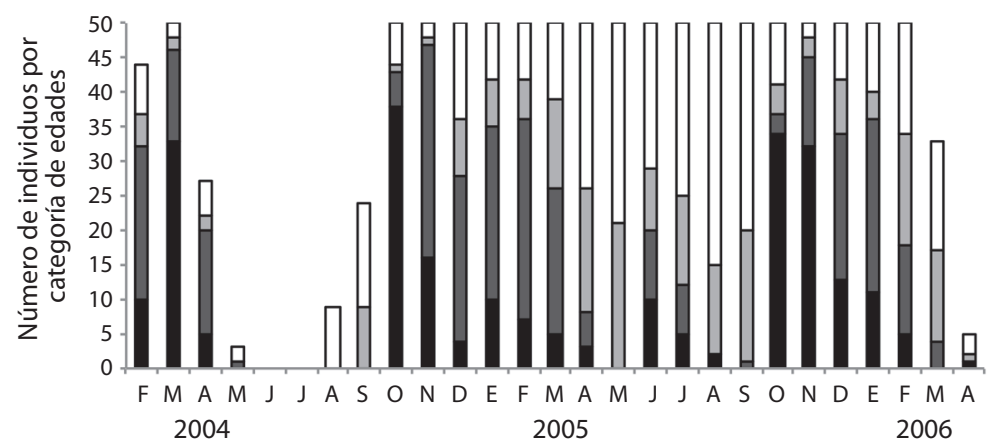

Fig. 2. (A) Biomasa de las categorías de edades de la población de Cornops aquaticum (mg de peso seco) y (B) abundancia de individuos por categoría de edades en Eichhornia crassipes durante el período de muestreo.

Fig. 2. (A) Biomass of Cornops aquaticum population age categories (mg of dry weight) and (B) abundance of individuals per age categories in Eichhornia crassipes during the sampling period.

posterior. El valor del ln de $a$ fue -9.66429 y el de $b 2.67537$ (Fig. 3).

Aplicabilidad de la longitud del fémur posterior para las estimaciones de biomasa poblacional de $C$. aquaticum: Los valores mensuales de biomasa poblacional del período enero a diciembre 2005 estimados mediante la ecuación del modelo de regresión lineal a partir de la longitud del fémur posterior (Fig. 4), no mostraron diferencias significativas con los obtenidos a partir del peso seco de los individuos de cada categoría de edades registrados para el mismo período (Fig. 1A y Fig. 2A) en la población de $C$. aquaticum asociada tanto a $E$. azurea $(\mathrm{p}=0.1484)$ como a $E$. crassipes $(\mathrm{p}=0.9906)$ (Fig. 4).

\section{DISCUSIÓN}

Las diferencias estacionales, anuales y entre plantas hospedadoras registradas en la biomasa de C. aquaticum de ambas lagunas, estarían determinadas por las variaciones en la abundancia y estructura poblacional de este insecto en E. azurea (Franceschini et al., 2005) y E. crassipes (Franceschini et al., 2013). Los mayores valores de biomasa se obtuvieron cuando la población estuvo dominada por adultos. Esta categoría de edad, junto con las ninfas B son las que presentaron los mayores valores de consumo diario de la población de C. aquaticum (Franceschini et al., 2013). Estos resultados son coincidentes con lo obtenido por Adis \& Junk (2003) y Braga et al. (2012) para 


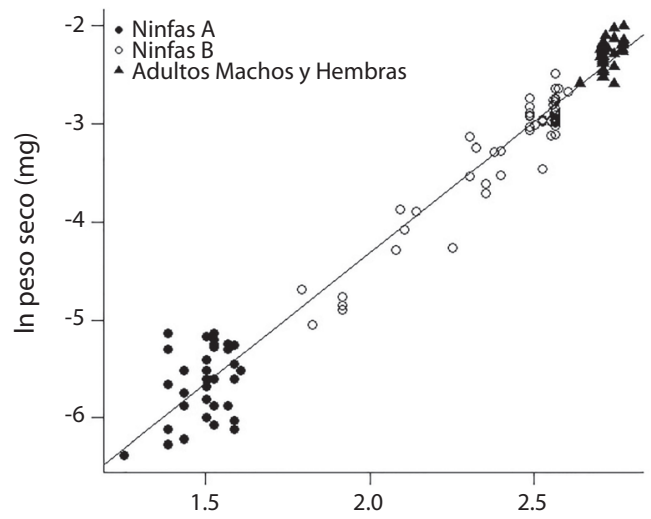

In longitud fémur posterior ( $\mathrm{mm}$ )

Fig. 3. Curva de regresión lineal obtenida a partir de la longitud del fémur posterior y el peso seco de los individuos de las diferentes categorías de edades de Cornops aquaticum.

Fig. 3. Lineal regression curve obtained from the hind femur length and the dry weight of Cornops aquaticum different age categories.
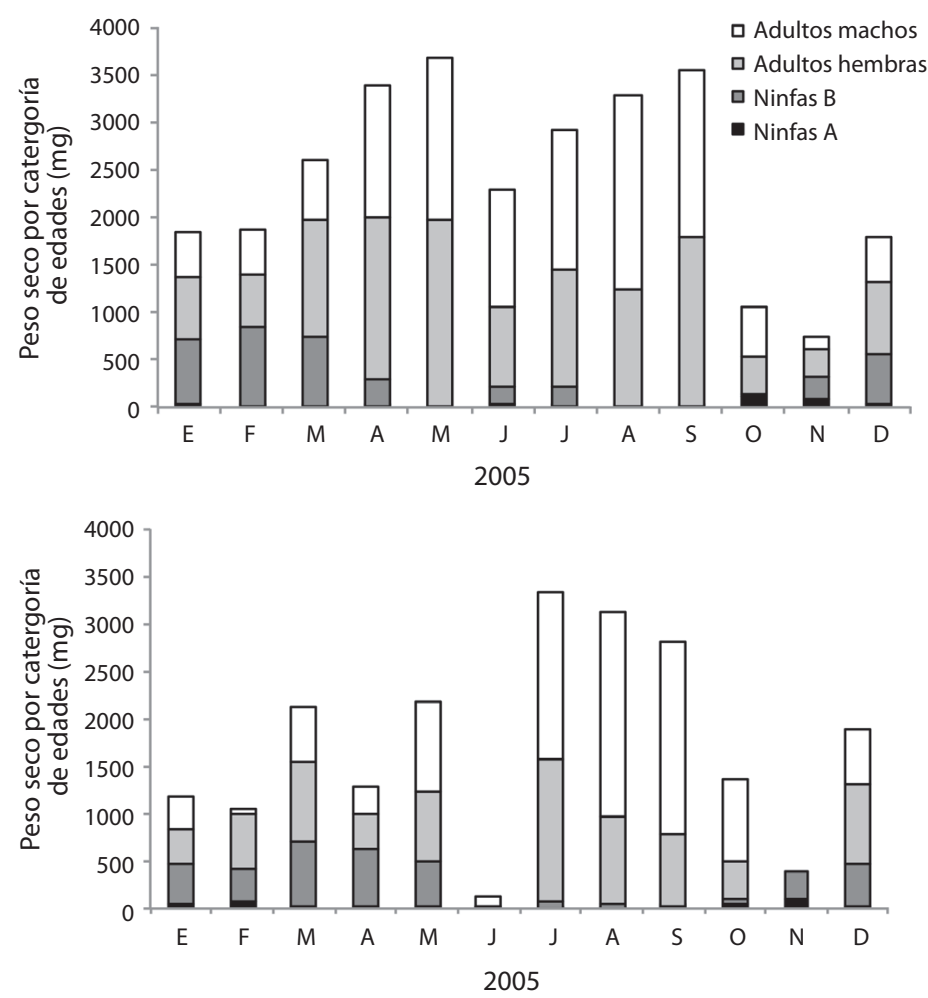

Fig. 4. Biomasa estimada de manera indirecta ( $\mathrm{mg}$ de peso seco) en las distintas categorías de edades de la población de Cornops aquaticum en 2005, en las praderas flotantes de Eichhornia azurea (A) y Eichhornia crassipes (B).

Fig. 4. Biomass estimated by indirect way ( $\mathrm{mg}$ of dry weight) on different age categories of Cornops aquaticum populations during 2005, in Eichhornia azurea (A) and Eichhornia crassipes (B) floating meadows. 
poblaciones de C. aquaticum en el Amazonas y por Silva et al. (2010b) en el Pantanal de Mato Grosso. Resultados diferentes se obtuvieron en el acridoideo Paulinia acuminata, De Geer, 1773 (Orthoptera: Acrididae), en el cual los picos de biomasa de la población estuvieron asociados a la mayor abundancia de ninfas (Vieira, \& Adis, 1992).

El peso seco de los individuos de C. aquaticum presentó alta variación entre las distintas categorías de edades. Esto parece ser característico de los insectos hemimetábolos que, por poseer el cuerpo completamente esclerotizado, la mayor parte del crecimiento e incremento de peso ocurre al momento de la muda cuando se produce la nueva cutícula blanda (Benke et al., 1999). El mismo autor manifiesta que existe menor variación del peso seco entre los estadios ninfales de los insectos hemimetábolos en comparación con los holometábolos. La mayor variabilidad del peso seco por individuo obtenido en la categoría de edad hembras adultas estaría determinada por los diferentes estados de maduración de los ovarios de las hembras de C. aquaticum.

La longitud corporal (B) ha sido ampliamente utilizada como medida de dimensión lineal para estimar la biomasa de los invertebrados (Hodar, 1996; Benke et al., 1999; Miserendino, 2001; Sabo et al., 2002). En $C$. aquaticum, la longitud del fémur posterior fue la medida más precisa para estimar la biomasa por individuo y poblacional. Esto coincide con lo expuesto por Franceschini et al. (2005) donde la longitud del fémur posterior presentó el menor coeficiente de variación y fue la medida que mejor separó los distintos estadios de C. aquaticum. Cepeda-Pizarro, Vega, Vásquez \& Elgueta (2003), afirman que la longitud del fémur posterior es uno de los caracteres morfométricos de menor variabilidad en el acridio Elasmoderus wagenknechti, Liebermann, 1954 (Orthoptera: Tristiridae).

El valor promedio de $b$ encontrado para la longitud corporal de los insectos de todos los órdenes, la mayoría de las familias y de muchas especies es $<3$, especialmente en los taxa acuáticos (Benke et al., 1999; Becker et al., 2009). Esto coincide con lo encontrado en este estudio, ya que el valor de $b$ obtenido para las poblaciones de $C$. aquaticum es cercano a 3 , siendo muy similar también al valor hallado por Hodar (1996) para el orden Orthoptera y por Sabo et al. (2002) para ortópteros terrestres. El valor de $b$ obtenido sugiere que el peso seco de los insectos depende en mayor medida de la superficie corporal que del volumen del cuerpo del individuo (Engelmann, 1961; Towers, Henderson, \& Veltman, 1994).

Varios autores sostienen que las ecuaciones obtenidas a nivel específico, presentan una mejor capacidad predictiva que las ecuaciones basadas en datos agrupados en niveles taxonómicos más altos (Smock, 1980; Hodar, 1996; Benke et al., 1999; Cressa, 1999a; Miserendino, 2001; Sabo et al., 2002). Nuestros resultados han sido obtenidos a partir de mediciones y pesos secos que abarcan todo el rango de tamaño de individuos de la población de C. aquaticum, lo cual es señalado por Hodar (1996) como un factor clave para la obtención de una buena ecuación de regresión.

La similitud entre la biomasa poblacional obtenida para el período enero a diciembre 2005 de manera indirecta a partir de la longitud del fémur posterior y la obtenida de manera directa con el peso seco de los individuos, refuerza los resultados obtenidos en las regresiones. Por lo tanto, el modelo de regresión lineal propuesto para C. aquaticum a partir de la longitud del fémur posterior, permite realizar estimaciones bastante precisas y confiables de la biomasa individual y poblacional de esta especie.

La longitud del fémur posterior, a diferencia de otras medidas de dimensión lineal, no es afectada por las técnicas de preservación del material y resulta fácil de registrar, agilizando el procesamiento de datos cuando se trabaja con poblaciones muy numerosas como la de este acridio. Debido a que es una medida precisa y poco subjetiva al momento de realizar la medición (Franceschini et al., 2005), resulta de gran utilidad para realizar estimaciones de biomasa de forma rápida y sencilla. Esto se debe a que es una estructura esclerotizada, sujeta a menor distorsión o posibilidades de 
ruptura ante la manipulación que la longitud corporal, coincidiendo con lo expuesto por Becker et al. (2009).

Las variaciones de biomasa por individuo y poblacional determinan el rol que cumple esta especie como herbívoro y como recurso alimentario para los depredadores en las redes tróficas de las praderas flotantes de E. azurea y E. crassipes. Los mayores valores de peso seco por individuo en hembras adultas y mayores valores de peso seco en ninfas de estadio VI con respecto a los adultos machos, explican la mayor capacidad de consumo de las hembras adultas de $C$. aquaticum y los valores similares de consumo por individuo que presentan los machos adultos y las ninfas B. Por otra parte, los diferentes valores de biomasa por individuo en $C$. aquaticum guardan relación con los depredadores que tiene este herbívoro para cada categoría de edades de la población. Entre éstos, Ludovix fasciatus, Gyllenhal, 1836 (Coleoptera: Curculionidae) se alimenta de los huevos de C. aquaticum, Phlugis teres, De Geer, 1773 (Orthoptera: Tettigoniidae) y Actinosoma pentacanthum, Walckenaer, 1842 (Araneae: Araneidae) depredan sobre las ninfas y, aves como Jacana jacana, Linnaeus, 1766 (Charadriiformes: Jacanidae), Zonotrichia capensis, Müller, 1776 (Passeriformes: Emberizidae) y algunas especies de anfibios consumen los adultos (Bennett, \& Zwölfer, 1968; Zwölfer, \& Bennett, 1969; Duré, \& Kehr, 2001, 2004; Duré, Schaefer, Hamann, \& Kehr, 2004; Alessio, Beltzer, Lajmanovich, \& Quiroga, 2005).

Finalmente, los resultados obtenidos muestran que existen importantes variaciones estacionales e interanuales en la biomasa poblacional de $C$. aquaticum, lo que lleva a confirmar la primera hipótesis planteada $(a)$. Además, los valores similares de biomasa poblacional obtenidos de manera directa (peso seco de los individuos) e indirecta (aplicando el modelo de regresión lineal propuesto), llevan a confirmar la segunda hipótesis planteada $(b)$ la cual sostiene que la biomasa por individuo y poblacional de $C$. aquaticum puede ser estimada indirectamente a partir de una medida de dimensión lineal.

\section{AGRADECIMIENTOS}

Este trabajo fue financiado por el HICWA Project (Host-Insect Coevolution on Waterhyacinth) dirigido por Joachim Adis del Tropical Ecology Working Group, Max-Planck Institute for Limnology (Alemania), durante el período 2004-2008 y por el Proyecto de Investigación Científica y Tecnológica (PICT) 2160-2011 del Fondo para la Investigación Científica y Tecnológica (FONCyT), Argentina.

\section{RESUMEN}

La estimación de la biomasa en las poblaciones de insectos, es un factor clave para cuantificar los recursos disponibles y los flujos de energía en las redes tróficas de los ecosistemas. Cornops aquaticum es un herbívoro común en las plantas de Eichhornia en los humedales del nordeste de Argentina. Nuestro objetivo fue analizar la variación de su biomasa en relación a las distintas categorías de edades de la población de este acridio, en dos plantas huésped: Eichhornia azurea y Eichhornia crassipes. Para ello, se realizaron muestreos estándar de las poblaciones de $C$. aquaticum con una red entomológica de $70 \mathrm{~cm}$ de diámetro, en dos humedales con E. azurea y $E$. crassipes en las provincias de Corrientes y Chaco; además, se obtuvo el peso seco de los individuos (de manera directa e indirecta) y, se propuso un modelo de regresión para estimar la biomasa de C. aquaticum de manera indirecta a partir de una medida de dimensión lineal (longitud del fémur posterior). Un total de 2307 individuos fueron recolectados y separados en distintas categorías de edades; se obtuvo su abundancia y distintas medidas de dimensión lineal. El modelo propuesto fue $\ln P S=\ln a+b^{*} \ln H$ (donde $\mathrm{PS}=$ peso seco, $a$ y $b$ son constantes y $\mathrm{H}=$ longitud del fémur posterior $)\left(\mathrm{R}^{2}=0.97\right)$. Las variaciones en la biomasa de las poblaciones de $C$. aquaticum se debieron a la abundancia relativa de cada categoría de edad y al peso seco individual de estos acridios. No hubo diferencias significativas entre la biomasa de las poblaciones de $C$. aquaticum obtenida por los métodos directo e indirecto en las praderas flotantes de E. azurea y E. crassipes. Este modelo facilita el cálculo de la biomasa individual y poblacional de C. aquaticum y acelera el procesamiento de un gran número de muestras. Finalmente, los valores altos de biomasa poblacional e individual de las categorías de edades (especialmente en adultos) enfatizan la importancia de C. aquaticum como consumidor y como recurso para los depredadores en las redes alimenticias de las praderas flotantes de Eichhornia.

Palabras clave: peso seco, Eichhornia, fémur, acridio semiacuático, modelo de regresión, abundancia poblacional, lirio de agua. 


\section{REFERENCIAS}

Adis, J., \& Junk, W. (2003). Feeding impact and bionomics of grasshopper Cornops aquaticum on the water hyacinth Eichhornia crassipes in the Central Amazonian floodplains. Studies in Neotropical Fauna and Environment, 38, 245-249.

Adis, J., Lhano, M., Hill, M., Junk, W., Marques, M. I., \& Oberholzer, H. (2004). What determines the number of juveniles instars in the tropical grasshopper Cornops aquaticum (Acrididae: Orthoptera)? Studies in Neotropical Fauna and Environment, 39, 127-132.

Adis, J., Bustorf, E., Lhano, M., Amedegnato, C., \& Nunes, A. L. (2007). Distribution of Cornops grasshoppers (Leptysminae: Acrididae: Orthoptera) in Latin America and the Caribbean Islands. Studies in Neotropical Fauna and Environment, 42, 11-24.

Alessio, V. G., Beltzer, A. H., Lajmanovich, R. C., \& Quiroga, M. A. (2005). Ecología alimentaria de algunas especies de Passeriformes (Furnariidae, Tyrannidae, Icteridae y Emberizidae): consideraciones sobre algunos aspectos del nicho ecológico. Insugeo Miscelánea, 14, 441-482.

Becker, B., Moretti, M. S., \& Castillo, M. (2009). Lengthdry mass relationships for a typical shredder in Brazilian streams (Trichoptera: Calamoceratidae). Aquatic Insects, 31, 227-234.

Benke, A. (1996). Secondary production of macroinvertebrates. In F. R. Hauer, \& G. A. Lamberti (Eds.), Methods in stream ecology (pp. 557-578). New York: Academic Press.

Benke, A. C., Huryn, A. D., Smok, L. A., \& Wallace, J. B. (1999). Length-mass relationships for freshwater macroinvertebrates in North America with particular reference to the southeastern United States. Journal of the North American Benthological Society, 18, 308-343.

Bennett, F. D., \& Zwölfer, H. (1968). Exploration for natural enemies of the water hyacinth in northern South America and Trinidad. Hyacinth Control Journal, 7, 44-52.

Braga, C. E., Nunes, A. L., Morais, J. W., \& Adis, J. (2012). Fenologia de Cornops aquaticum (Bruner 1906) (Orthoptera: Acrididae) associado à Eichhornia crassipes (Mart.) Solms (Pontederiaceae) em um lago de Várzea na Amazônia Central, Brasil. Revista de la Sociedad Entomológica Argentina, 70, 185-196.

Brede, E. G., Adis, J., \& Schneider, P. (2007). What is responsible for the variance in life history traits of South American semi-aquatic grasshopper (Cornops aquaticum)? A test of three possible hypotheses. Studies in Neotropical Fauna and Environment, 42, 225-233.

Bruniard, E. D. (1981). El clima de las planicies del Norte Argentino. Vol. I-II. Resistencia, Argentina: Editorial de la Facultad de Humanidades de la Universidad Nacional del Nordeste.

Bruniard, E. D. (1996). Geografia de los climas y de las formaciones vegetales: Las zonas térmicas y la vegetación natural. Corrientes, Argentina: Editorial de la Universidad Nacional del Nordeste.

Carbonell, C. S. (2001). The grasshopper tribe Phaeoparini (Acridoidea: Romaleidae). Publications on Orthopteran Diversity. The Orthopterist's Society, 1-48.

Center, T. D., Hill, M. P., Cordo, H., \& Julien, M. H. (2002). Waterhyacinth. In R. Van Driesche, B. Blossey, M. Hoddle, S. Lyon, \& R. Reardon (Eds.), Biological Control of invasive plants in the eastern United States (pp.41-64). West Virginia, USA, Forest Health and Technology Enterprises Team.

Cepeda-Pizarro, J., Vega, S., Vásquez, H., \& Elgueta, M. (2003). Morfometría y dimorfismo sexual de Elasmoderus wagenknechti (Liebermann) (Orthoptera: Tristiridae) en dos eventos de irrupción poblacional. Revista Chilena de Historia Natural, 76, 417-435.

Cigliano, M. M., De Wysiecki, M. L., \& Lange, C. (2000). Grasshopper (Orthoptera) species diversity in the Pampas, Argentina. Diversity and Distributions, 6, 81-91.

Cressa, C. (1999a). Dry mass estimates of some tropical aquatic insects. Revista de Biología Tropical, 47, 133-141.

Cressa, C. (1999b). Dry mass estimation of tropical aquatic insects using different short-term preservation methods. Revista de Biología Tropical, 47, 143-149.

Da Silva, C. J., \& Esteves, F. A. (1993). Biomass of three macrophytes in the Pantanal of the Mato Grosso, Brasil. Journal of Ecology and Environmental Sciences, 19, 11-23.

Duré, M. I., \& Kehr, A. I. (2001). Differential exploration of trophic resource by two Pseudid frogs from Corrientes, Argentina. Journal of Herpetology, 35, 340-343.

Duré, M. I., \& Kehr, A. I. (2004). Influence of microhabitat on the trophic ecology of two Leptodactylids from Northeastern Argentina. Herpetologica, 60, 295-303.

Duré, M. I., Schaefer, E. F., Hamann, M. I., \& Kehr, A. I. (2004). Consideraciones ecológicas sobre la dieta, la reproducción y el parasitismo de Pseudopaludicola boliviana (Anura: Leptodactylidae) de Corrientes, Argentina. Phyllomedusa, 3, 121-131.

Engelmann, M. D. (1961). The role of soil arthropods in the energetics of an old field community. Ecological Monographs, 31, 221-238.

Franceschini, M. C., Capello, S., Lhano, M., Adis, J., \& De Wysiecki, M. L. (2005). Morfometría de los estadíos ninfales Cornops aquaticum Bruner (1906) (Acrididae: Leptysminae) en Argentina. Amazoniana, 18, 373-386. 
Franceschini, M. C., Adis J., Poi de Neiff, A., \& De Wysiecki, M. L. (2007). Fenología de Cornops aquaticum Bruner (Orthoptera: Acrididae: Leptysminae) en un camalotal de Eichhornia azurea (Pontederiaceae) en Argentina. Amazoniana, 19, 149-158.

Franceschini, M. C., De Wysiecki, M. L., Poi de Neiff, A., Galassi, M. E., \& Martínez, F. S. (2011a). The role of the host-specific grasshopper Cornops aquaticum (Orthoptera: Acrididae) as consumer of native Eichhornia crassipes (Pontederiaceae) floating meadows. Revista de Biología Tropical, 59, 1407-1418.

Franceschini, M. C., Neiff, J. J., \& Amedegnato, C. (2011b). The Oxycaryum cubense floating mat as refuge of Cornops aquaticum (Orthoptera: Acrididae), a new control agent of the water hyacinth. Studies in Neotropical Fauna and Environment, 46, 203-210.

Franceschini, M. C., De Wysiecki, M. L., \& Poi, A. (2013). Age structure and feeding of the Neotropical grasshopper Cornops aquaticum (Bruner) (Orthoptera: Acrididae) on the waterhyacinth. Neotropical Entomology, 42, 344-350

Gardner, K. T., \& Thompson, D. C. (2001). Development and phenology of the beneficial grasshopper Hesperotettix viridis. Southwestern Entomologists, 26, 305-313.

Hodar, J. A. (1996). The use of regression equations for estimation of arthropod biomass in ecological studies. Acta Oecologica, 17, 421-433.

Infostat (Version 1.1). (2002). Córdoba, Argentina: Grupo InfoStat Universidad Nacional de Córdoba.

Jannot, J. A., Ko, E., Herrmann, D. L., Skinner, L., Butzen, E., Akman, O., \& Juliano, S. (2009). Densitydependent polyphenism and geographic variation in size among two populations of lubber grasshoppers (Romalea microptera). Ecological Entomology, 34, 644-651.

Johnson, D. L. (1989). The effects of timing and frequency of application of Nosema locustae (Microspora: Microsporida) on the infection rate and activity of grasshoppers (Orthoptera: Acrididae). Journal of Invertebrate Pathology, 54, 353-362.

Lhano, M. G., Adis, J., Marques, M. I., \& Battirola, L. D. (2005). Cornops aquaticum (Orthoptera, Acrididae, Leptysminae): aceitação de plantas alimentares por ninfas vivendo em Eichhornia azurea (Pontederiaceae) no Pantanal Norte, Brasil. Amazoniana, 18, 397-404.

Martínez, S. F., Franceschini, M. C., \& Poi, A. (2013). Preferencia alimentaria de Neochetina eichhorniae Warner (Coleoptera: Curculionidae) por plantas acuáticas de diferente valor nutritivo bajo condiciones naturales (Corrientes Argentina). Revista Colombiana de Entomología, 39, 81-87.
Messi, J., Kekeunou, S., \& Weise, S. (2006). Abundance and life cycle of Zonocerus variegatus (Orthoptera: Pyrgomorphidae) in the humid forest zone of Southern Cameroon. Entomological Science, 9, 23-30.

Miserendino, M. L. (2001). Length-mass relationships for macroinvertebrates in freshwater environments of Patagonia (Argentina). Ecología Austral, 11, 3-8.

Neiff, J. J, Casco, S., \& Poi de Neiff, A. (2008). Response of Eichhornia crassipes (Pontederiaceae) to water level fluctuations in two lakes with different connectivity in the Paraná River floodplain. Revista de Biología Tropical, 56, 613-623.

Nunes, A. L., Adis, J., \& Nunes de Mello, J. A. S. (1992). Estudo sobre o ciclo de vida e fenologia de Stenacris fissicauda fissicauda (Bruner 1908) (Orthoptera: Acrididae) em um lago de várzea da Amazônia Central, Brasil. Boletim do Museu Paraense Emílio Goeldi, Série Zoologia, 8, 349-374.

Nunes, A. L., \& Adis, J. (1994). Comportamento populacional de Tucayaca gracilis (Giglio-Tos 1897) frente a oscilação do nível d' água na várzea da Amazônia Central. Boletim do Museu Paraense Emílio Goeldi, Série Zoologia, 10, 211-224.

Piedade, M. T. F., \& Junk, W. J. (2000). Natural Grasslands and Herbaceous plants in the Amazon Floodplain and their Use. In W. J. Junk, J. J. Ohly, M. T. F. Piedade, \& M. G. M. Soares (Eds.), The Central Amazon Floodplain: Actual Use and Options for a Sustainable Management (pp. 269-290). Leiden, The Netherlands: Backhuys Publishers.

RStudio (Version 0.97.248). (2013). The RFAQ. Retrieved form $h$ ttp://CRAN.R project.org/doc/FAQ/R-FAQ. html

Sabo, J. L., Bastow, J. L., \& Power, M. E. (2002). Lengthmass relationships for adult aquatic and terrestrial invertebrates in a California watershed. Journal of the North American Benthoogical Society, 21, 336-343.

Silva, F. R., Marques, M. I., Battirola, L. D., \& Lhano, M. G. (2010a). Fenologia de Cornops aquaticum (Bruner) (Orthoptera: Acrididae) in Eichhornia azurea (Pontederiaceae) no Norte do Pantanal de Mato Grosso. Neotropical Entomology, 39, 535-542.

Silva, F. R., Marques, M. I., Battirola, L. D., \& Lhano, M. G. (2010b). Variação do peso fresco em Cornops aquaticum (Bruner) (Orthoptera: Acrididae) associado a Eichhornia azurea (Sw) Kunth (Pontederiaceae) em uma baía no Pantanal de Poconé, Mato Grosso. Revista Brasileira de Entomologia, 54, 91-95.

Smock, L. A. (1980). Relationships between body size and biomass of aquatic insects. Freshwater Biology, 10, 375-383. 
Squitier, J. M., \& Capinera, J. (2002). Observations of the phenology of common Florida grasshoppers (Orthoptera: Acrididae). Florida Entomological Society, 85, 227-234.

Torrusio, S., Cigliano, M. M., \& De Wysiecki, M. L. (2002). Grasshopper (Orthoptera: Acrididae) and plant community relationships in the Argentine Pampas. Journal of Biogeography, 29, 221-229.

Towers, D. J., Henderson, I. M., \& Veltman, C. J. (1994). Predicting dry weight of New Zealand aquatic macroinvertebrates from linear dimensions. New
Zealand Journal of Marine and Freshwater Research, 28, 159-166.

Vieira, M. F., \& Adis, J. (1992). Abundância y biomassa de Paulinia acuminata (De Geer, 1773) (Orthoptera, Pauliniidae) em um lago de várzea da Amazônia Central. Amazoniana, 12, 337-352.

Zwölfer, H., \& Bennett, F. D. (1969). Ludovix fasciatus GYLL. (Col. Curculionidae), an entomophagous weevil. Entomologist's Monthly Magazine, 105, 122-123. 